\title{
A Comparison of Dentin Tubule Occlusion Properties of Dipotassium Oxalate against Potassium Nitrate: An In Vitro Study
}

\author{
Viet $\operatorname{Tran}^{1}$, Thanhphuong N Dinh ${ }^{2}$, Thomas YH Yoon ${ }^{3}$
}

\begin{abstract}
Aim: The aim of this study is to compare the efficacy of dipotassium oxalate and potassium nitrate to occlude dentinal tubules.

Materials and methods: This study utilized Parkinson model of longitudinal dentin tubule occluding properties of dentifrices under a 4-day acid challenge. Dentin disks of approximately $1.5 \mathrm{~mm}$ thick were sectioned from the crowns of the freshly extracted molars. The disks were randomized into three sets of 15 and treated with dipotassium oxalate, potassium nitrate, or used as a control. The disks were then subjected to a 4-day acid challenge and evaluated by scanning electron microscopy (SEM).

Results: On days 1, 2, and 3, dipotassium oxalate showed significant occlusion of dentinal tubules. On day 4, no significant difference was observed between dipotassium oxalate and potassium nitrate. Both test groups showed better occlusion properties in comparison to the control. Conclusion: Through the use of a 4-day acid challenge, this study demonstrates that both agents can indeed occlude dentinal tubules. Initially, dipotassium oxalate does occlude dentinal tubules faster than potassium nitrate. However, at the conclusion of the acid challenge, minimal differences were observed in occlusion rate among the two agents. Further studies should be conducted to determine the efficacy of these two agents.

Clinical significance: Both dipotassium oxalate and potassium nitrate can help treat patients with dentinal hypersensitivity.

Keywords: Dentin desensitizing agent, Dentin hypersensitivity, Dipotassium oxalate, Potassium nitrate.

The Journal of Contemporary Dental Practice (2020): 10.5005/jp-journals-10024-2754
\end{abstract}

\section{INTRODUCTION}

Dentin hypersensitivity $(\mathrm{DH})$ is defined as transient tooth pain due to exposed dentin tubules. It is a well-known oral health concern that affects more than $43 \%$ of the adult population. ${ }^{1}$ Individuals affected with $\mathrm{DH}$ are known to change their daily habits to alleviate the symptoms. Schiff et al. note that DH patients will modify their habits by eliminating certain foods and drinks from their customary diet. Of greater concern, these patients also tend to become noncompliant with daily oral hygiene measures. Consequently, $\mathrm{DH}$ patients may have a higher rate of caries formation, gingival inflammation, and possibly periodontal issues. ${ }^{2}$

The most widely accepted theory about $\mathrm{DH}$ is Brannstrom hydrodynamic theory, which states that the pain is due to "abrupt movement of fluid within the dentinal tubules in response to cold and heat (thermal), osmotic (sweets), mechanical (chewing) and evaporative stimuli (air blasts)." ${ }^{3}$ Dentin is composed of canaliculi tubules that, under normal conditions, are isolated from the external environment by enamel or cementum. For DH development, dentin, along with their canaliculi, must become exposed to the oral cavity due to gingival recession; loss of enamel/cementum by erosion, abrasion, attrition abfraction; or by incorrect oral hygiene technique. ${ }^{4}$ Furthermore, the cold, osmotic, and evaporative stimuli cause an outward flow of fluids, while mechanical and hot stimuli cause an inward flow. The movement of fluid leads to mechanical deformation and activation of A fiber located near the odontoblasts.

Treatment for hypersensitivity generally involves finding mechanisms to desensitize the nerves or occluding the tubules that lead to reduce the flow of fluid. ${ }^{5}$ Two popular commercially available products that show promise in reducing sensitivity are
${ }^{1}$ VA Medical Center, Orlando, Florida, USA

2,3 3 Lake Erie College of Osteopathic Medicine, School of Dental Medicine, Bradenton, Florida, USA

Corresponding Author: Thomas YH Yoon, Lake Erie College of Osteopathic Medicine, School of Dental Medicine, Bradenton, Florida, USA, Phone: +1 941405 1506, e-mail: tyoon@lecom.edu

How to cite this article: Tran V, Dinh TN, Yoon TYH. A Comparison of Dentin Tubule Occlusion Properties of Dipotassium Oxalate against Potassium Nitrate: An In Vitro Study. J Contemp Dent Pract 2020;21(2):119-123.

Source of support: This study was generously funded by a grant from the Lake Erie College of Osteopathic Medicine, School of Dental Medicine, Bradenton, Florida, USA

Conflict of interest: None

dipotassium oxalate and potassium nitrate. Dipotassium oxalate has been theorized to reduce fluid flow by forming precipitates within dentinal tubules that block fluid flow. One benefit of oxalates is that it is relatively insoluble in acid.

Conversely potassium ions, typically in the form of potassium nitrate, modify the nerve impulse by decreasing the excitability of the $A$ fibers that surround the base of the odontoblastic process. This results in a reduction in tooth sensitivity. Although extensively studied, the effectiveness of potassium nitrate-containing dentifrices in treating $\mathrm{DH}$ is not clear. ${ }^{6}$

Various mechanisms have been utilized to determine the effectiveness of antisensitivity agents. One study used to assess the ability of various materials to occlude dentinal tubules is the 4-day 
occlusion model. ${ }^{7}$ This model utilizes prolonged soaks in whole human saliva and acid challenges to recreate the oral environment. The use of this model in conjunction with scanning electron microscopy (SEM) of the treated surface may show evidence to support the treatment.

The purpose of this study is to use the 4-day occlusion model to compare the effectiveness of dipotassium oxalate and potassium nitrate. The null hypothesis is that no differences are observed between the dipotassium oxalate and the potassium nitrate experimental groups.

\section{Materials and Methods}

The study was approved by the Institutional Review Board of Lake Erie College of Osteopathic Medicine (protocol 24-136) on March 13, 2017.

One hundred and six sound extracted human third molars were obtained anonymously and stored in a water/sodium hypochlorite solution (1:10). The third molars were extracted to prevent future problems due to unfavorable orientation or uneruption. Dentin disks of approximately $1.5 \mathrm{~mm}$ thick were sectioned from the crowns of the molars and randomized into three sets of 15 for a total sample size of 45 (Fig. 1). Each set was then mounted into monoacrylic and polished sequentially with $800,1,200$, and finally 2,500 grit paper. ${ }^{8}$ The mounted disks were then rinsed with EDTA ( $\left.\mathrm{p} K_{\mathrm{a}}: 1.782\right)$ and sonicated for 2 minutes to remove the smear layer and reveal open tubules that run perpendicular to the polished surface.

The specimens were mounted on a metal peg and prepared for SEM (JEOL). Dentin specimen were individually imaged at $1,400 \times$ magnification without sputter coating to provide a baseline measure of tubule density and appearance for each disk specimen prior to start of study. All 45 samples were imaged by SEM. Five set areas of each dentin specimen were viewed to ensure evaluation of the dentin. One representative image of each specimen was recorded. Steps were performed as scheduled (Table 1). All samples were evaluated by a single examiner.

This study utilized Parkinson model of longitudinal dentin tubule occluding properties of dentifrices, ${ }^{7}$ which consisted of 4 days of twice daily application of test materials and twice daily acid challenge on days 3 and 4 . One set of dentin disk was set aside as a control group and received no treatment. One set of dentin disk was treated with dipotassium oxalate strips (Crest Sensi-Stop Strips; Proctor and Gamble, Cincinnati, $\mathrm{OH}$, USA). The dipotassium

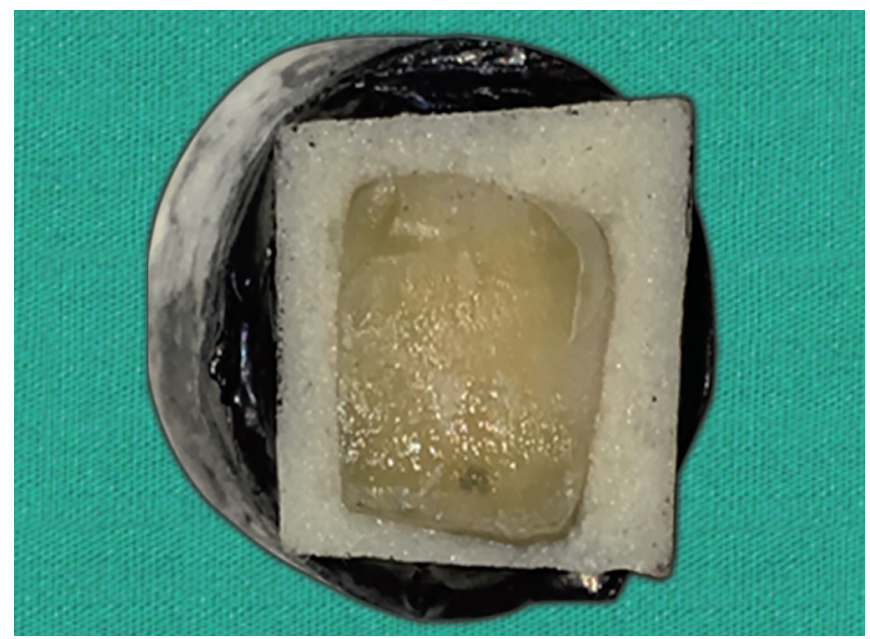

Fig. 1: Example of prepared dentin disk oxalate strips were cut to size and placed on the dentin disk for 10 minutes and removed. A third set of dentin disks were treated with potassium nitrate (Sensodyne; GalaxoSmithKline, Brentford, United Kingdom). The potassium nitrate was manually brushed by a single person for 60 seconds on each disk using a manual toothbrush. The dentin disks were then rested in the slurry for 10 seconds and gently rinsed with deionized water. All samples were then returned to $20 \mathrm{~mL}$ of fresh whole human saliva donated by a single individual.

An acid challenge was given 60 minutes posttreatment on days 3 and 4 by placing the dentin specimens into unstirred orange juice ( $\mathrm{pH}$ of 3.35) for 5 minutes and then gently rinsing in deionizing water before returning to $20 \mathrm{~mL}$ to new fresh whole human saliva. At the end of the day, the samples were removed, dried, and examined via SEM. After SEM analysis, the disks were returned to the saliva.

Visual grading of the extent of tubule occlusion was made by ranking the amount of occlusion from the SEM images per the 1-5 scale used in Parkinson model, ${ }^{7}$ where the score of 5 represents completely occluded; 4 is mostly occluded; 3 is equal amounts of occlusion and nonocclusion; 2 is mostly unoccluded; and 1 completely unoccluded tubules. Samples were rated in tenths of a point based upon the examiner evaluation. A single examiner was used to evaluate all the samples. Occlusion grades were analyzed using the analysis of variance for all samples separately for each day. Comparisons between different treatment groups were presented along with $95 \%$ confidence intervals.

\section{Results}

The initial dentin samples had an abundance of open tubules. After day 1, a fine layer of deposit can be seen on the SEM and the original tubules became partially occluded mostly on the two test groups. The dipotassium oxalate group showed the highest rate of initial occlusion (mean value 3.883). The potassium nitrate group did show occluding properties (mean value 3.200) but was not superior to the dipotassium oxalate group. The control group showed minimal occluding properties (mean value 0.900 ). Both the test groups delivered significantly greater dentinal occlusion scores compared to the control ( $p<0.0001)$.

On day 2, the dentin tubules for both test groups showed some change, with potassium nitrate showing a slightly greater increase in occlusion score. With each day, more fine minerals were deposited into the dentin tubules of the potassium nitrate dentin disks. The dipotassium oxalate group showed the highest rate of initial occlusion (mean value 3.950). The potassium nitrate group did show occluding properties (mean value 3.450 ) but was not superior to the dipotassium oxalate group. The control group showed a slight increase in dentinal occlusion (1.1133). Both the test groups delivered significantly greater dentinal occlusion scores compared to the control $(p<0.0001)$.

On day 3 , the dipotassium oxalate group continued to show the highest rate of initial occlusion (mean value 4.033). The potassium nitrate group did show occluding properties (mean value 3.633) but was not superior to the dipotassium oxalate group $(p=0.25)$. The control group showed an increase in dentinal occlusion (1.383). Both the test groups delivered significantly greater dentinal occlusion scores compared to the control $(p<0.0001)$.

On day 4, improvements were seen in both experimental groups, with a significant improvement in the potassium nitrate group (mean value 4.233). A slight increase was seen in the dipotassium oxalate group (mean value 4.233). The control also demonstrated slight improvement (mean value 1.450). Significant 
Table 1: Schedule for the 4-day dentin disk model and acid challenge. All times \pm 30 minutes

\begin{tabular}{|c|c|c|c|c|c|c|c|}
\hline & 800 & 900 & 1,100 & 1,130 & 1,430 & 1,530 & 1,630 \\
\hline Disinfection of dentin disks & $\mathrm{X}$ & & & & & & \\
\hline Disks immersed in saliva & & $x$ & & & & & \\
\hline Treatment applied and disks returned to saliva & & & $\mathrm{X}$ & & $\mathrm{x}$ & & \\
\hline Disks immersed in orange for 5 minutes (days 3-4) & & & & $\mathrm{X}$ & & $\mathrm{X}$ & \\
\hline $\begin{array}{l}\text { Disks removed from saliva, rinsed, and stored in humid } \\
\text { container prior to scanning }\end{array}$ & & & & & & & $x$ \\
\hline
\end{tabular}

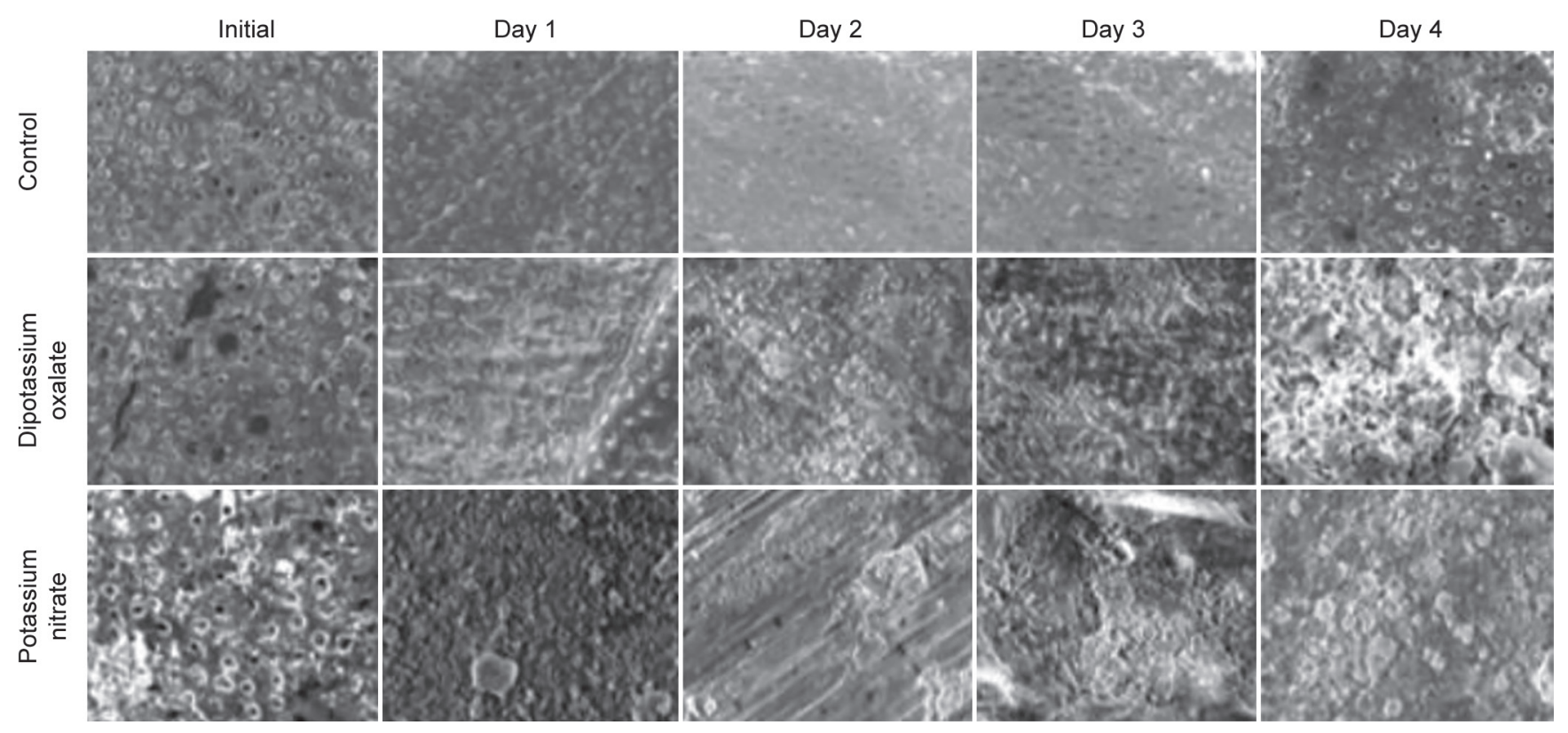

Fig. 2: Scanning electron microscopy images of dentin disks during the 4-day acid challenge

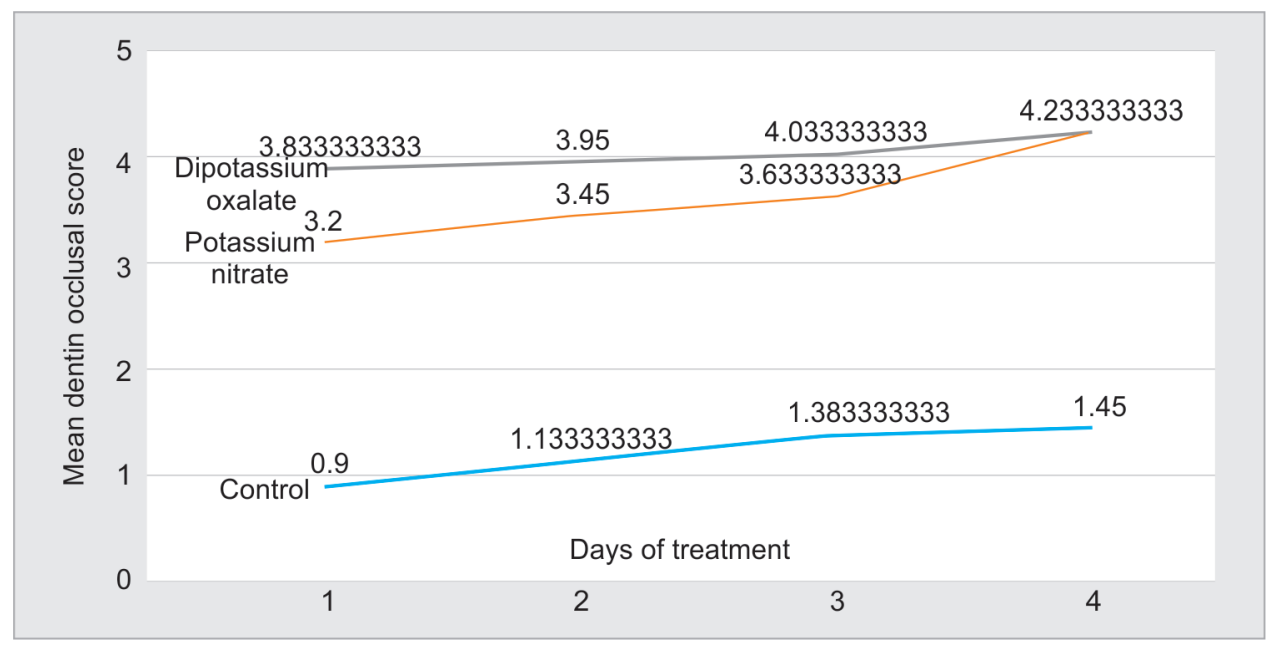

Fig. 3: Mean dentinal occlusal scores over the treatment period

difference was observed between the test groups in comparison to the control ( $p<0.0001)$. No statistical significance was observed between the test groups $(p=1.000)$. The progression of dentinal occlusion is shown in Figures 2 and 3.

\section{Discussion}

The clinical effectiveness of treating $\mathrm{DH}$ has been repeatedly demonstrated. ${ }^{9,10}$ The occlusion of dentinal tubules has been shown to be an effective mechanism to decrease the overall DH. Dentin tubule occlusion can be achieved by the deposition of an occluding layer on top of the dentin or by the introduction of an occluding material into the dentine tubule. ${ }^{11}$ The two active ingredients that were utilized in this study were dipotassium oxalate and potassium nitrate.

Since the late 1970s, it is common to treat DH using oxalates. Numerous in vitro studies show significant decrease in hydraulic conductance on dentin disks that are treated with oxalates. ${ }^{12,13}$ 
Recent in vivo studies also show the effectiveness of oxalates to treat DH. Lynch et al. compared the use of potassium oxalate mouth rinse in comparison to a placebo in a large multicenter study. Their results show that patients had a higher rate of thermal sensitivity control within the potassium oxalate group vs the control $(69.3 \%$ test vs $44.6 \%$ control). ${ }^{14}$

A significant advantage to the utilization of oxalates is their relative insolubility in acid. ${ }^{15}$ Greater resistance of a $\mathrm{DH}$ agent to acid increases the deposition rate, thus occluding the dentin tubule at a faster rate. Eliades et al. compared several commercial mouth rinses and their ability to occlude dentin tubules in the presence of an acid challenge. Their results show that a $1.4 \%$ potassium oxalate mouth rinse had the greatest resistance to acid dissolution. ${ }^{16}$ The current literature supports our finding that dipotassium oxalate has better resistance to an acid challenge and can occlude dentin tubules effectively and faster compared to potassium nitrate.

Historically, studies have proved that potassium nitrate is a safe and effective means of reducing $\mathrm{DH} .{ }^{17,18}$ Previous research has demonstrated that potassium nitrate reduces $\mathrm{DH}$ by increasing the extracellular potassium ion concentrations. If high concentration of potassium ions are sustained over time, the synapse between nerve cells becomes blocked and the tooth experiences less hypersensitivity. ${ }^{19}$ Because an increase in potassium ions is needed over time, studies have shown that it can take upward of 4 weeks for potassium nitrate to show efficacy. ${ }^{20}$

Recently, potassium nitrate's mechanism of action has been subject to debate. Recent research has shown that potassium nitrate has the ability to block dentinal tubules, similar to potassium oxalate. Pinto et al. utilized SEM and energy dispersive X-rays on rodent teeth treated with potassium nitrate. Their study revealed that potassium nitrate could partially obstruct dentinal tubules after 4 days of daily application. ${ }^{21}$ James et al. utilized extracted human molars and subjected them to toothpaste and mouthwash that contained potassium nitrate. Through SEM analysis, they were able to show that human dentinal tubules show occlusion within 3 days of application. ${ }^{22}$ The findings of the SEM analysis are consistent with the current research. Future studies should be conducted to further explore dentinal occlusion properties of potassium nitrate.

A limitation of this study is the relative short time that the samples were subjected to an acid challenge. Since most DH medicaments are utilized for a long period of time, a subsequent study with an extended acid challenge would be beneficial. A second limitation of this study involves the location in which the sections of dentin were created. Mjör et al. demonstrated that marked differences were observed in the density of dentinal tubules from the upper section of the root in comparison to the apex of the tooth. ${ }^{23}$ Subsequent studies should utilize the same section of a tooth to ensure uniform distribution of dentinal tubule density.

\section{Conclusion}

Forty-three percent of individuals experienced some form of dentinal hypersensitivity. Potassium nitrate and dipotassium oxalate have been theorized to decrease dentinal hypersensitivity by occluding open tubules. Through the use of a 4-day acid challenge, this study demonstrates that both agents can indeed occlude dentinal tubules. Initially, dipotassium oxalate does occlude dentinal tubules faster than potassium nitrate. However, at the conclusion of the acid challenge, minimal differences were observed in occlusion rate among the two agents. Further studies should be conducted to determine the efficacy of these two agents.

\section{Clinical Significance}

Since dentinal hypersensitivity is a common clinical concern, it is important to investigate the efficacy of commercially available products. Dipotassium oxalate and potassium nitrate are two agents that are currently used in dental products designed to reduce hypersensitivity. This research reveals that both dipotassium oxalate and potassium nitrate can help treat patients with dentinal hypersensitivity by occluding dentinal tubules.

\section{ACKnOWLedgments}

The authors would like to thank the LECOM School of Dental Medicine Research Committee for their assistance with editing. The authors would also like to thank LECOM IPAAR for their assistance with statistical analysis.

\section{References}

1. Saeki K, Marshall GW, Gansky SA, et al. Strontium effects on root dentin tubule occlusion and nanomechanical properties. Dent Mater 2016;32(2):240-251. DOI: 10.1016/j.dental.2015.11.020.

2. Schiff T, Delgado E, Zhang Y, et al. Clinical evaluation of the efficacy of an in-office desensitizing paste containing $8 \%$ arginine and calcium carbonate in providing instant and lasting relief of dentin hypersensitivity. Am J Dent 2009;22(Spec No. A):8A-15A.

3. Brännström $M$, Lindén $L$, Aström $A$. The hydrodynamics of the dental tubule and of pulp fluid. Caries Res 1967;1(4):310-317. DOI: 10.1159/000259530.

4. Jacobsen PL, Bruce G. Clinical dentin hypersensitivity: understanding the causes and prescribing a treatment. J Contemp Dent Pract 2001;2(1):1-12. DOI: 10.5005/jcdp-2-1-27.

5. Nagata $\mathrm{T}$, Ishida $\mathrm{H}$, Shinohara $\mathrm{H}$, et al. Clinical evaluation of a potassium nitrate dentifrice for the treatment of dentinal hypersensitivity. J Clin Periodontol 1994;21(3):217-221. DOI: 10.1111/j.1600-051X.1994. tb00307.x.

6. Poulsen S, Errboe M, Hovgaard O, et al. Potassium nitrate toothpaste for dentine hypersensitivity. Cochrane Database Syst Rev 2001;2:CD001476. DOI: 10.1002/14651858.CD001476.

7. Parkinson CR, Willson RJ. A comparative in vitro study investigating the occlusion and mineralization properties of commercial toothpastes in a four day dentin disc model. J Clin Dent 2011;22(3):74-81.

8. Burnett $G$, Willson R, Lucas R. In vitro studies investigating the dentin tubule-occlusion properties of an experimental anhydrous stannous fluoride dentifrice. Am J Dent 2013;26(Spec No A):10A-14A.

9. Parkinson $C R$, Hughes $N$, Jeffrey $P$, et al. The efficacy of a dentifrice containing $0.454 \% \mathrm{w} / \mathrm{w}$ stannous fluoride in providing relief from the pain of dentine hypersensitivity: an 8 week clinical study. Am J Dent 2013;26(Spec No A):25A-31A.

10. Schiff T, Zhang YP, DeVizio W, et al. A randomized clinical trial of the desensitizing efficacy of three dentifrices. Compend Contin Educ Dent Suppl 2000;27:4-10.

11. Arnold WH, Prange M, Naumova EA. Effectiveness of various toothpastes on dentine tubule occlusion. J Dent 2015;43(4):440-449. DOI: 10.1016/j.jdent.2015.01.014.

12. Pashley DH, O'Meara JA, Kepler EE, et al. Dentin permeability. Effects of desensitizing dentifrices in vitro. J Periodontol 1984;55(9):522-525. DOI: 10.1902/jop.1984.55.9.522.

13. Greenhill JD, Pashley DH. The effects of desensitizing agents on the hydraulic conductance of human dentin in vitro. J Dent Res 1981;60(3):686-698. DOI: 10.1177/00220345810600030401.

14. Lynch MC, Perfekt R, McGuire JA, et al. Potassium oxalate mouthrinse reduces dentinal hypersensitivity: a randomized controlled clinical study. J Am Dent Assoc 2018;149(7):608-618. DOI: 10.1016/ j.adaj.2018.02.027.

15. Pereira JC, Segala AD, Gillam DG. Effect of desensitizing agents on the hydraulic conductance of human dentin subjected to different 
surface pre-treatments--an in vitro study. Dent Mater 2005;21(2):129_ 138. DOI: 10.1016/j.dental.2004.02.007.

16. Eliades G, Mantzourani M, Labella R, et al. Interactions of dentine desensitisers with human dentine: morphology and composition. J Dent 2013;41(Suppl 4):S28-S39. DOI: 10.1016/S0300-5712(13)70004-6.

17. Clark DC, Hanley JA, Geoghegan S, et al. Effectiveness of a fluoride varnish and a desensitizing toothpaste in treating dentinal hypersensitivity. J Periodontol Res 1985;20(2):212-219. DOI: 10.1111/ j.1600-0765.1985.tb00428.x.

18. Tarbet WJ, Buckner A, Stark MM, et al. The pulpal effects of brushing with a 5 percent potassium nitrate paste used for desensitization. Oral Surg Oral Med Oral Pathol 1981;51(6):600-602. DOI: 10.1016/ S0030-4220(81)80009-6.

19. Orchardson R, Gillam DG. The efficacy of potassium salts as agents for treating dentin hypersensitivity. J Orofac Pain 2000;14(1):9-19.
20. Addy $M$, Mostafa P, Newcombe R. Dentine hypersensitivity: a comparison of five toothpastes used during a 6-week treatment period. Br Dent J 1987;163(2):45-51. DOI: 10.1038/sj.bdj. 4806185.

21. Pinto SC, Silveira CM, Pochapski MT, et al. Effect of desensitizing toothpastes on dentin. Braz Oral Res 2012;26(5):410-417. DOI: 10.1590/ S1806-83242012000500006.

22. James JM, Puranik MP, Sowmya KR. Dentinal tubule occluding effect of potassium nitrate in varied forms, frequencies and duration: an in vitro SEM analysis. J Clin Diagn Res 2017;11(8):ZC06-ZC08. DOI: 10.7860/JCDR/2017/26442.10340.

23. Mjör IA, Nordahl I. The density and branching of dentinal tubules in human teeth. Arch Oral Biol 1996;41(5):401-412. DOI: 10.1016/00039969(96)00008-8. 